\title{
Author Correction: Generalization guides human exploration in vast decision spaces
}

\author{
Charley M. Wu (D), Eric Schulz (D), Maarten Speekenbrink (D), Jonathan D. Nelson (1) and Björn Meder(D)
}

Correction to: Nature Human Behaviour https://doi.org/10.1038/s41562-018-0467-4, published online 12 November 2018.

In the version of this article initially published, there was a coding error that required updating the simulated learning curves in Fig. $3 \mathrm{~b}$ and a math error that required updating Eq. 1 in the main text and Eq. 6 in the Supporting Information. The conclusions and all statistical tests reported in the paper remain unaffected.

The first error was a mistake in the code used to produce the simulated learning curves in Fig. 3b (the middle two graphs) for Experiment 2. Specifically, the simulations for the smooth environment were mistakenly run using the rewards from the rough environment. Thus, the difference in performance between the original and corrected versions can be attributed to a mismatch between the environment and participant parameter estimates used to specify the models.

The learning curves were only used to provide qualitative assessments in the article, and do not affect any of the analyses or statistical tests. Nevertheless, the corrected learning curves provide even stronger evidence for the main conclusions, by showing a closer correspondence between the function learning model (green line) and human performance (pink line). The publicly available code repository has been updated to reflect this correction (https://github.com/charleywu/gridsearch) and the authors thank B. Klein and C. Riedl for directing attention to this issue.

The second error was a typo in the equation for the radial basis function (RBF) kernel, which was presented as Eq. 1 in the main text and as Eq. 6 in the Supporting Information. In all cases, the analyses, reported parameter estimates, and the generative model of rewards used in experiments, were with respect to the correct version of the equation. The errors have been corrected in the HTML and PDF versions of the article.

Original :

$k_{\mathrm{RBF}}(\mathbf{x}, \mathbf{x} \prime)=\exp \left(-\frac{\|\mathbf{x}-\mathbf{x} /\|^{2}}{\lambda}\right)$

Corrected :

$k_{\mathrm{RBF}}(\mathbf{x}, \mathbf{x} \prime)=\exp \left(-\frac{\|\mathbf{x}-\mathbf{x}\|^{2}}{2 \lambda^{2}}\right)$
Original

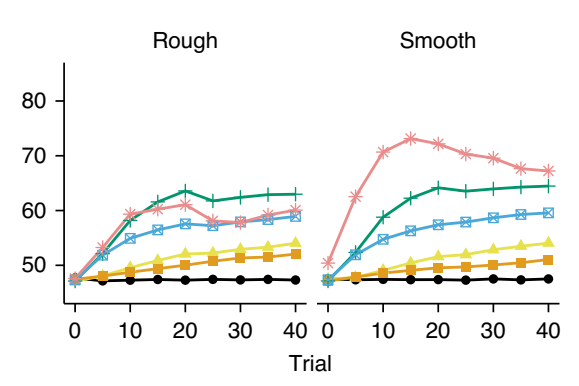

Corrected

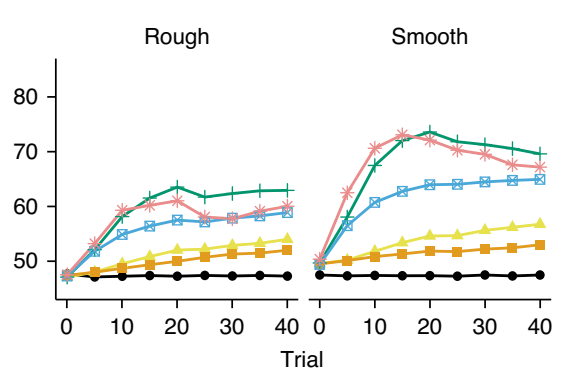

Fig. 3 | Original and Corrected.

Published online: 23 October 2020

https://doi.org/10.1038/s41562-020-00958-w

(C) The Author(s), under exclusive licence to Springer Nature Limited 2020 